\title{
Grunsky 算子
}

\author{
沈玉良
}

(苏州大学数学系, 苏州 215006)

摘要讨论了单叶函数 Grunsky 算子的全纯依赖性及紧性.

关键词 单叶函数 拟共形映射 Grunsky 算子 紧算子 Hilbert-Schmidt 算子

$\operatorname{MSC}(2000)$ 主题分类 $30 \mathrm{C} 62,30 \mathrm{C} 55,47 \mathrm{~B} 07$

\section{1 引言}

从单叶函数理论中一些熟知的结果开始 ${ }^{[1]}$. 对于 $\Delta^{*}=\{z:|z|>1\} \cup\{\infty\}$ 上的亚纯函 数 $f(z)=z+\sum_{n=1}^{\infty} a_{n} z^{-n}$, 它的 Grunsky 系数 $\alpha_{m n}(f)$ 定义如下:

$$
\log \frac{f(z)-f(\zeta)}{z-\zeta}=-\sum_{m, n=1}^{\infty} \alpha_{m n}(f) z^{-m} \zeta^{-n}
$$

其中当 $z=\zeta=\infty$ 时, 对数分支取值为 0 . 于是 $f$ 在 $\Delta^{*}$ 中单叶, 即 $f \in \Sigma_{0}$, 当且仅当以下不 等式对所有 $x=\left(x_{1}, x_{2}, \ldots\right) \in l^{2}$ 成立:

$$
\left|\sum_{m, n=1}^{\infty} \sqrt{m n} \alpha_{m n}(f) x_{m} x_{n}\right| \leqslant \sum_{m=1}^{\infty}\left|x_{m}\right|^{2} .
$$

如果 $f \in \Sigma_{0}$ 能够 $k$ - 拟共形延拓到单位圆 $\Delta=\{z:|z|<1\}$ 内, 即 $f \in \Sigma(k)$, 它的 Grunsky 系 数 $\alpha_{m n}(f)$ 满足更强的不等式

$$
\left|\sum_{m, n=1}^{\infty} \sqrt{m n} \alpha_{m n}(f) x_{m} x_{n}\right| \leqslant k \sum_{m=1}^{\infty}\left|x_{m}\right|^{2} .
$$

反之, 从 (1.3) 式可以知道存在 $k^{\prime} \geqslant k$, 使得 $f$ 能够 $k^{\prime}$ - 拟共形延拓到单位圆 $\Delta=\{z:|z|<1\}$ 内, 即 $f \in \Sigma\left(k^{\prime}\right)$.

我们用泛函分析的语言来重新叙述上述结果. 首先 $l^{2}$ 是序列 $x=\left(x_{m}\right)$ 组成的 Hilbert 空间, 内积和范数分别是

$$
\langle x, y\rangle=\sum_{m=1}^{\infty} x_{m} \bar{y}_{m}, \quad\|x\|=\left(\sum_{m=1}^{\infty}\left|x_{m}\right|^{2}\right)^{\frac{1}{2}} .
$$

给定 $f \in \Sigma_{0}$, 根据下式定义 Grunsky 算子 $G(f): l^{2} \rightarrow l^{2}$ :

$$
G(f):\left(x_{m}\right) \mapsto\left(\sum_{n=1}^{\infty} \sqrt{m n} \alpha_{m n}(f) x_{n}\right) .
$$

收稿日期: 2007-05-23; 接受日期: 2007-07-16

新世纪优秀人才支持计划 (批准号: NCET-06-0504) 资助项目

E-mail: ylshen@suda.edu.cn 
于是

$$
\langle G(f) x, \bar{x}\rangle=\sum_{m, n=1}^{\infty} \sqrt{m n} \alpha_{m n}(f) x_{m} x_{n} .
$$

由于 $\alpha_{m n}(f)=\alpha_{n m}(f), G(f)$ 的共轭算子 $G^{*}(f)$ 满足 $G^{*}(f) \bar{x}=\overline{G(f) x}$. (1.2) 和 (1.5) 式说明 $|\langle G(f) x, \bar{x}\rangle| \leqslant\|x\|^{2}$, 由于 $\alpha_{m n}(f)=\alpha_{n m}(f)$, 这等价于 $\|G(f) x\| \leqslant\|x\|$, 因此 $G(f)$ 是一个有界 线性算子, 且 $\|G(f)\| \leqslant 1$. (1.3) 式及其逆和 (1.5) 式说明, $\|G(f)\|<1$ 当且仅当 $f \in \cup_{k<1} \Sigma(k)$.

众所周知, Grunsky 算子在单叶函数理论 ${ }^{[1]}$ 和 Teichmüller 空间 ${ }^{[2-15]}$ 研究中都起着 十分重要的作用. 本文将讨论 Grunsky 算子对单叶函数的依赖性. 我们主要讨论 $G(f)$ 对 $f \in \cup_{k<1} \Sigma(k)$ 的全纯依赖性, 还将讨论 Grunsky 算子的紧性.

\section{2 万有 Teichmüller 空间}

在本节中, 将介绍一些本文要用到的基本概念和记号 ${ }^{[16-18]}$.

记 $M$ 是 $\Delta$ 上有界函数空间 $L^{\infty}(\Delta)$ 中的单位球. 对于 $\mu \in M$, 记 $f_{\mu}$ 是扩充复平 面 $\overline{\mathbb{C}}$ 上的拟共形映射, 它在 $\Delta$ 内以 $\mu$ 为复特征, 在 $\Delta^{*}=\overline{\mathbb{C}}-\bar{\Delta}$ 共形, 且当 $z \rightarrow \infty$ 时, $f_{\mu}(z)=z+O\left(|z|^{-1}\right) . \mu$ 和 $\nu$ 称为是等价的, 如果 $f_{\mu}\left|\Delta^{*}=f_{\nu}\right| \Delta^{*} . \mu$ 的等价类记为 $[\mu]$. 所有 等价类的集合 $T$ 称为万有 Teichmüller 空间. $T$ 中两点 $\left[\mu_{1}\right]$ 和 $\left[\mu_{2}\right]$ 之间的 Teichmüller 距离 定义为

$$
\tau\left(\left[\mu_{1}\right],\left[\mu_{2}\right]\right)=\inf \left\{\frac{1}{2} \log \frac{1+\left\|\frac{\nu_{1}-\nu_{2}}{1-\bar{\nu}_{2} \nu_{2}}\right\|_{\infty}}{1-\left\|\frac{\nu_{1}-\nu_{2}}{1-\bar{\nu}_{1} \nu_{2}}\right\|_{\infty}}:\left[\nu_{1}\right]=\left[\mu_{1}\right],\left[\nu_{2}\right]=\left[\mu_{2}\right]\right\} .
$$

万有 Teichmüller 空间 $T$ 有另外一个重要的描述. 记 $B$ 是 $\Delta^{*}$ 上的全纯函数 $\varphi$ 组成的 Banach 空间, 范数定义为

$$
\|\varphi\|_{B}=\sup _{z \in \Delta^{*}}|\varphi(z)|\left(|z|^{2}-1\right)^{2} .
$$

考虑映射 $\Psi: M \rightarrow B, \Psi(\mu)=S\left(f_{\mu} \mid \Delta^{*}\right)$, 其中 $S(f)$ 表示扩充复平面 $\overline{\mathbb{C}}$ 上一个局部单叶函数 $f$ 的 Schwarz 导数, 即 $S(f)=\left(f^{\prime \prime} / f^{\prime}\right)^{\prime}-1 / 2\left(f^{\prime \prime} / f^{\prime}\right)^{2}$. $\Psi$ 是一个全纯浸入, 且它在 0 处的微分 有如下表达式:

$$
d_{0} \Psi(\nu)(z)=-\frac{6}{\pi} \iint_{\Delta} \frac{\nu(\zeta)}{(\zeta-z)^{4}} d \xi d \eta .
$$

$\Psi$ 诱导了一个 1-1 映射 $\beta: T \rightarrow B$, 称为 Bers 嵌入. 通过 Bers 嵌入, $T$ 上存在一个自然的复 结构使得自然投影 $\Phi: M \rightarrow T$ 是一个全纯浸入, 而 $\beta$ 是 $T$ 上的双全纯同构.

$f \in \Sigma_{0}$ 称为是渐近共形的, 如果 $f$ 可以拟共形延拓到单位圆 $\Delta$ 内, 使得当 $|z| \rightarrow 1_{-}$时, $f$ 的复特征 $\mu=\partial_{\bar{z}} f / \partial_{z} f$ 满足 $\mu(z) \rightarrow 0$. 渐近共形映射有许多等价定义, 而且有许多从经 典复分析出发的研究 ${ }^{[19]}$, 另外在渐近 Teichmüller 空间的研究中起着重要的作用 ${ }^{[16,20-23]}$. 称 $f \in \Sigma_{0}$ 属于 Weil-Petersson 类, 如果 $f$ 可以拟共形延拓到单位圆 $\Delta$ 内, 并且它的复特征 $\mu=\partial_{\bar{z}} f / \partial_{z} f$ 在 Poincaré 度量下平方可积, 即

$$
\iint_{\Delta}|\mu(z)|^{2}\left(1-|z|^{2}\right)^{-2} d x d y<\infty .
$$

Weil-Petersson 类映射在万有 Teichmüller 空间的 Weil-Petersson 几何研究中有着重要的作 用. 一个很重要的事实是 Weil-Petersson 类映射是渐近共形的 ${ }^{[24]}$. 
记 $L_{0}$ 是 $L^{\infty}(\Delta)$ 中当 $|z| \rightarrow 1_{-}$时 $\mu(z) \rightarrow 0$ 的函数 $\mu$ 组成的闭子空间, 而 $B_{0}$ 是 $B$ 中当 $|z| \rightarrow 1_{+}$时 $\phi(z)\left(|z|^{2}-1\right)^{2} \rightarrow 0$ 的函数 $\phi$ 组成的闭子空间. 记 $M_{0}=M \cap L_{0}$, 则 $T_{0}=\Phi\left(M_{0}\right)$ 是 $T$ 的闭子流形, 且 $\beta\left(T_{0}\right)=\beta(T) \cap B_{0}$. 特别地, $f \in \cup_{k<1} \Sigma(k)$ 是渐近共形的当且仅当 $S(f) \in B_{0}$.

我们需要 Weil-Petersson 类映射的一个对应结果 ${ }^{[24]}$. 记 $L_{2}$ 是 $L^{\infty}(\Delta)$ 中满足下列条件 的函数 $\mu$,

$$
\|\mu\|_{2}^{2}=\iint_{\Delta}|\mu(z)|^{2}\left(1-|z|^{2}\right)^{-2} d x d y<\infty,
$$

并记 $B_{2}$ 是 $B$ 中满足下列条件的函数 $\phi$,

$$
\|\phi\|_{2}^{2}=\iint_{\Delta^{*}}|\phi(z)|^{2}\left(|z|^{2}-1\right)^{2} d x d y<\infty .
$$

记 $M_{2}=M \cap L_{2}$, 则 $T_{2}=\Phi\left(M_{2}\right)$ 满足 $\beta\left(T_{2}\right)=\beta(T) \cap B_{2}$, 即 $f \in \cup_{k<1} \Sigma(k)$ 属于 Weil-Petersson 类当且仅当 $S(f) \in B_{2}$.

注 $1 \quad B_{2}$ 是一个复 Hilbert 空间, 内积为

$$
\langle\phi, \psi\rangle=\iint_{\Delta^{*}} \phi(z) \bar{\psi}(z)\left(|z|^{2}-1\right)^{2} d x d y,
$$

这恰好是万有 Teichmüller 空间上的 Weil-Petersson 度量.

\section{Grunsky 映射的全纯性}

本节讨论 $G(f)$ 关于 $f \in \Sigma_{0}$ 的依赖性. 事实上, 主要讨论在 $\cup_{k<1} \Sigma(k)$ 上的全纯依赖性. 根据定义, Teichmüller 空间 $T$ 中的每一点 $[\mu]$ 确定了 $\cup_{k<1} \Sigma(k)$ 中唯一的映射 $f_{\mu} \mid \Delta^{*}$. 我们根 据 $G([\mu])=G\left(f_{\mu} \mid \Delta^{*}\right)$ 来定义 Grunsky 映射. (1.3) 式蕴涵 $\|G([\mu])\| \leqslant \tanh \tau([0],[\mu])$. 我们将 证明 $G$ 是 $T$ 上的全纯映射.

首先介绍一些无穷维全纯性的基本结果. 设 $X$ 和 $Y$ 是两个复 Banach 空间, $U$ 是 $X$ 的一个非空开子集. 映射 $f: U \rightarrow Y$ 称为是全纯的, 如果 $f$ 是局部有界的, 且对所有的 $(u, x) \in(U, X), f$ 存在复 Gateaux 导数

$$
d_{u} f(x)=\lim _{t \rightarrow 0} \frac{f(u+t x)-f(u)}{t} .
$$

关于无穷维全纯性有许多等价定义 ${ }^{[25,26]}$. 下面要提到的等价定义将把 Banach 空间之 间映射的全纯性转化为平面上复值函数的全纯性. 我们需要考虑 $Y$ 的对偶空间 $Y^{*}$. 对于 $Y^{*}$ 的子集 $A$, 记

$$
A^{\perp}=\left\{y \in Y: y^{*}(y)=0, y^{*} \in A\right\} .
$$

$A$ 满足 $A^{\perp}=\{0\}$ 时称为是整体的. 根据文献 [27](也见文献 [28]), $Y^{*}$ 的子集 $A$ 称为是可决 定的, 如果存在常数 $C$, 使得对所有的 $y \in Y$,

我们有以下结果 ${ }^{[25-28]}$.

$$
\|y\| \leqslant C \sup \left\{\frac{\left|y^{*}(y)\right|}{\left\|y^{*}\right\|}: y^{*} \in A-\{0\}\right\} .
$$

命题 3.1 映射 $f: U \rightarrow Y$ 是全纯的, 当且仅当它满足下述条件之一:

(1) $f$ 是局部有界的, 且对所有的 $(u, x) \in(U, X)$, 映射 $t \rightarrow f(u+t x)$ 是复平面 $\mathbb{C}$ 上零点 的一个邻域到 $Y$ 的全纯映射; 
(2) $f$ 是连续的, 且存在 $Y^{*}$ 的一个整体子集 $A$, 使得对所有的 $y^{*} \in A$, 函数 $y^{*} \circ f: U \rightarrow \mathbb{C}$ 是全纯的;

(3) $f$ 是局部有界的, 且存在 $Y^{*}$ 的一个可决定子集 $A$, 使得对所有的 $y^{*} \in A$, 函数 $y^{*} \circ f: U \rightarrow \mathbb{C}$ 是全纯的.

我们来证明 $G$ 是 $T$ 上的全纯映射. 任给 $[\mu] \in T, G([\mu])=G\left(f_{\mu} \mid \Delta^{*}\right)$ 是 $l^{2}$ 到自身的有 界线性算子. 记 $L\left(l^{2}\right)$ 是 $l^{2}$ 到自身的有界线性算子组成的空间, 于是 $G$ 是从万有 Teichmüller 空间 $T$ 到 $L\left(l^{2}\right)$ 的有界映射.

定理 $1 \quad G: T \rightarrow L\left(l^{2}\right)$ 是全纯映射.

证明 由于 $\Phi: M \rightarrow T$ 是全纯浸入, 故只需证明 $\tilde{G}=G \circ \Phi$ 是 $M$ 上的全纯映射. 由于 $\tilde{G}$ 的有界性, 根据命题 3.1 只需证明存在 $L\left(l^{2}\right)$ 的对偶空间 $L^{*}\left(l^{2}\right)$ 中的一个可决定子集 $A$, 使 得对任意的 $\beta \in A$, 函数 $\beta \circ \tilde{G}: M \rightarrow \mathbb{C}$ 是全纯的.

首先来找一个 $L^{*}\left(l^{2}\right)$ 的整体子集. 记 $e_{k}=\left(x_{m}\right) \in l^{2}$, 其中 $x_{k}=1$, 而 $m \neq k$ 时, $x_{m}=0$. 于是 $e_{1}, e_{2}, \ldots, e_{k}, \ldots$ 是 $l^{2}$ 的一组基. 对于每一对整数 $(k, l)$, 定义 $\beta_{k l} \in L^{*}\left(l^{2}\right)$ 为

$$
\beta_{k l}(L)=\left\langle L e_{k}, e_{l}\right\rangle, \quad L \in L\left(l^{2}\right) .
$$

记 $A_{0}=\left\{\beta_{k l}: k, l=1,2,3, \ldots\right\}$, 于是 $A_{0}^{\perp}=\{0\}$. 对于每一对整数 $(k, l)$ 和每一个 $\mu \in M$,

$$
\beta_{k l} \circ \tilde{G}(\mu)=\left\langle G\left(f_{\mu} \mid \Delta^{*}\right) e_{k}, e_{l}\right\rangle=\sqrt{k l} \alpha_{k l}\left(f_{\mu} \mid \Delta^{*}\right) .
$$

继续找 $L^{*}\left(l^{2}\right)$ 的可决定子集. 记 $l_{0}^{2}$ 是 $l^{2}$ 的由 $e_{1}, e_{2}, \ldots, e_{k}, \ldots$ 张成的子空间, 即 $l_{0}^{2}$ 是 $l^{2}$ 的所有序列 $x=\left(x_{m}\right)$ 的子空间, 其中项 $x_{m}$ 除了有限项以外均为零. 于是 $l_{0}^{2}$ 在 $l^{2}$ 中稠密. 对每一对 $(x, y), x, y \in l_{0}^{2}$, 定义 $\beta_{x, y} \in L^{*}\left(l^{2}\right)$ 为

$$
\beta_{x, y}(L)=\langle L x, y\rangle, \quad L \in L\left(l^{2}\right) .
$$

记 $A=\left\{\beta_{x, y}: x, y \in l_{0}^{2}\right\}$. 由于 $l_{0}^{2}$ 在 $l^{2}$ 中稠密, $A$ 是 $L^{*}\left(l^{2}\right)$ 的一个可决定子集. 对每一对 $(x, y), x, y \in l_{0}^{2}$, 和每一个 $\mu \in M$,

$$
\beta_{x, y} \circ \tilde{G}(\mu)=\left\langle G\left(f_{\mu} \mid \Delta^{*}\right) x, y\right\rangle=\sum_{m, n=1}^{\infty} \sqrt{m n} \alpha_{m n}\left(f_{\mu} \mid \Delta^{*}\right) x_{m} \bar{y}_{n} .
$$

由于 $x_{m}$ 和 $y_{n}$ 中除了有限项以外都是零, $\beta_{x, y} \circ \tilde{G}$ 的全纯性由以下结论推出:

子定理 Grunsky 系数是万有 Teichmüller 空间 $T$ 上的全纯函数.

证明 为简单起见, 记 $\alpha_{m n}\left(f_{\mu} \mid \Delta^{*}\right)=\alpha_{m n}(\mu)$. 由命题 3.1, 需要证明对每一对 $(m, n)$ 和 $(\mu, \nu) \in\left(M, L^{\infty}(\Delta)\right), \alpha_{m n}(\mu+t \nu)$ 在 $\mathbb{C}$ 上零点的某邻域内全纯. 为此, 取 $r>0$, 使得 $\|\mu\|_{\infty}+r\|\nu\|_{\infty}<1$, 并记 $\Delta_{r}=\{z \in \mathbb{C}:|z|<r\}$. 对于 $(t, u, v) \in \Delta_{r} \times \Delta \times \Delta$, 当 $u v=0$ 时记 $H(t, u, v)=0$, 而当 $u v \neq 0$ 时记

$$
H(t, u, v)=\log \frac{f_{\mu+t \nu}\left(u^{-1}\right)-f_{\mu+t \nu}\left(v^{-1}\right)}{u^{-1}-v^{-1}} .
$$

固定 $t, H$ 是 $u$ 和 $v$ 的全纯函数, 固定 $u$ 和 $v, H$ 是 $t$ 的全纯函数. 由著名的 Hartogs 定 理 ${ }^{[29], ~} H$ 是全纯的, 并且在 $\Delta_{r} \times \Delta \times \Delta$ 中有收敛的 Maclaurin 展式

$$
H(t, u, v)=\sum_{l=0}^{\infty} \sum_{m, n=1}^{\infty} c_{l m n} t^{l} u^{m} v^{n} .
$$

在上述级数中不存在求和次序问题, 因而对每一对 $(m, n)$,

$$
\alpha_{m n}(\mu+t \nu)=-\sum_{l=0}^{\infty} c_{l m n} t^{l}, \quad t \in \Delta_{r} .
$$


这完成了子定理和定理的证明.

注 2 Krushkal (见文献 [2], p. 303) 曾不加证明地断言 Grunsky 映射的全纯性, 并且在 他以后的研究中反复运用 ${ }^{[3-11]}$, 而 Shiga-Tanigawa (见文献 [14], p. 363) 则不加证明地指出 了 Grunsky 系数的全纯性. 由于 Grunsky 算子在 Teichmüller 空间的研究中有着十分重要的 应用 ${ }^{[2-15]}$, 我们给出了这些结果的完整证明. Takhtajan-Teo ${ }^{[30]}$ 考虑了一个类似于 Grunsky 映射的映射, 并证明它是全纯的.

在本节余下部分, 为了以后的应用计算 $\tilde{G}$ 在零点处的微分. 首先根据拟共形映射的表示 定理来计算 Grunsky 系数的微分.

以下关于拟共形映射的变分公式是熟知的 ${ }^{[18,31]}$ :

$$
f_{t \nu}(z)=z-\frac{t}{\pi} \iint_{\Delta} \frac{\nu(w)}{w-z} d u d v+o(t), \quad t \rightarrow 0 .
$$

于是, 当 $t \rightarrow 0$,

$$
\begin{aligned}
\log \frac{f_{t \nu}(z)-f_{t \nu}(\zeta)}{z-\zeta} & =-\frac{t}{\pi} \iint_{\Delta} \frac{\nu(w)}{(w-z)(w-\zeta)} d u d v+o(t) \\
& =-\frac{t}{\pi} \iint_{\Delta} \nu(w)\left(\sum_{m, n=1}^{\infty} z^{-m} \zeta^{-n} w^{m+n+2}\right) d u d v+o(t) \\
& =-\frac{t}{\pi} \sum_{m, n=1}^{\infty}\left(\iint_{\Delta} \nu(w) w^{m+n+2} d u d v\right) z^{-m} \zeta^{-n}+o(t),
\end{aligned}
$$

因此, 当 $t \rightarrow 0$,

$$
\sum_{m, n=1}^{\infty} \alpha_{m n}(t \nu) z^{-m} \zeta^{-n}=\frac{t}{\pi} \sum_{m, n=1}^{\infty}\left(\iint_{\Delta} \nu(w) w^{m+n+2} d u d v\right) z^{-m} \zeta^{-n}+o(t),
$$

由此得到

$$
d_{0} \alpha_{m n}(\nu)=\frac{1}{\pi} \iint_{\Delta} \nu(z) z^{m+n-2} d x d y .
$$

(3.1) 式曾在文献 $[3,4]$ 和文献 [14] 中出现过.

继续计算 $\tilde{G}$ 在零点处的微分. 我们断言

$$
d_{0} \tilde{G}(\nu):\left(x_{m}\right) \mapsto\left(\frac{1}{\pi} \sum_{n=1}^{\infty} \iint_{\Delta} \nu(z)\left(\sqrt{m n} x_{n} z^{m+n-2}\right) d x d y\right) .
$$

事实上, 如果用 $L_{0} \in L\left(l^{2}\right)$ 表示由 (3.2) 式确定的算子, 则

$$
\beta_{k l} \circ L_{0}=\left\langle L_{0} e_{k}, e_{l}\right\rangle=\frac{\sqrt{k l}}{\pi} \iint_{\Delta} \nu(z) z^{k+l-2} d x d y=\sqrt{k l} d_{0} \alpha_{k l}(\nu) .
$$

另一方面, 由于 $\beta_{k l} \circ \tilde{G}=\sqrt{k l} \alpha_{k l}$, 因此 $\beta_{k l} \circ d_{0} \tilde{G}(\nu)=\sqrt{k l} d_{0} \alpha_{k l}(\nu)$. 于是, $\beta_{k l} \circ d_{0} \tilde{G}(\nu)=\beta_{k l} \circ L_{0}$. 由于 $A_{0}^{\perp}=\{0\}$, 故得到 $d_{0} \tilde{G}(\nu)=L_{0}$, 因此 (3.2) 式成立.

接下来说明 $d_{0} \tilde{G}$ 的范数等于 1 , 证明的细节将在第 5 节中得到应用. 借用文献 $[3,4]$ 的 一些讨论. 对 $x=\left(x_{m}\right) \in l^{2}$ 定义

$$
\phi_{x}(z)=\frac{1}{\pi} \sum_{m, n=1}^{\infty} \sqrt{m n} x_{m} x_{n} z^{m+n-2}, \quad z \in \Delta .
$$

$\phi_{x}$ 在 $\Delta$ 中全纯, 且 $\iint_{\Delta}\left|\phi_{x}(z)\right| d x d y=\|x\|^{2}$.

$$
\left\langle d_{0} \tilde{G}(\nu) x, \bar{x}\right\rangle=\frac{1}{\pi} \sum_{m, n=1}^{\infty} \iint_{\Delta} \nu(z)\left(\sqrt{m n} x_{m} x_{n} z^{m+n-2}\right) d x d y
$$




$$
=\iint_{\Delta} \nu(z) \phi_{x}(z) d x d y .
$$

于是, $\left|\left\langle d_{0} \tilde{G}(\nu) x, \bar{x}\right\rangle\right| \leqslant\|\nu\|_{\infty}\|x\|^{2}$, 利用对称性可以说明 $\left\|d_{0} \tilde{G}(\nu) x\right\| \leqslant\|\nu\|_{\infty}\|x\|$. 于是 $\left\|d_{0} \tilde{G}(\nu)\right\|$ $\leqslant\|\nu\|_{\infty}$, 且 $\left\|d_{0} \tilde{G}\right\| \leqslant 1$. 另一方面, 对 $x \in l^{2}$, 记 $\nu_{x}=\left|\phi_{x}\right| / \phi_{x}$, 则 $\left\|\nu_{x}\right\|_{\infty}=1$. 于是

$$
\left|\left\langle d_{0} \tilde{G}\left(\nu_{x}\right) x, \bar{x}\right\rangle\right|=\iint_{\Delta}\left|\phi_{x}(z)\right| d x d y=\|x\|^{2},
$$

因此, $\left\|d_{0} \tilde{G}\left(\nu_{x}\right) x\right\|=\|x\|$, 且 $\left\|d_{0} \tilde{G}\left(\nu_{x}\right)\right\|=1=\left\|\nu_{x}\right\|_{\infty},\left\|d_{0} \tilde{G}\right\|=1$.

\section{Grunsky 算子的紧性}

本节讨论当 $f \in \cup_{k<1} \Sigma(k)$ 时 Grunsky 算子 $G(f)$ 的紧性. 主要结论是

定理 2 对 $f \in \cup_{k<1} \Sigma(k), G(f): l^{2} \rightarrow l^{2}$ 是一个紧算子当且仅当 $f$ 是渐近共形的.

证明 假设 $G(f): l^{2} \rightarrow l^{2}$ 是一个紧算子. 需要证明 $f$ 是渐近共形的. 我们来证明 $S(f) \in B_{0}$. 对 (1.1) 式的两边关于 $z$ 和 $\zeta$ 求微分可以得到

$$
\frac{f^{\prime}(z) f^{\prime}(\zeta)}{[f(z)-f(\zeta)]^{2}}-\frac{1}{(z-\zeta)^{2}}=-\sum_{m, n=1}^{\infty} m n \alpha_{m n}(f) z^{-(m+1)} \zeta^{-(n+1)} .
$$

让 $\zeta \rightarrow z$ 可以得到

$$
S(f)(z)=-6 \sum_{m, n=1}^{\infty} m n \alpha_{m n}(f) z^{-(m+n+2)} .
$$

对 $z \in \Delta^{*}$, 记 $x_{z}=\left(x_{m}\right)$, 其中 $x_{m}=\frac{|z|^{2}-1}{|z|} \cdot \frac{\sqrt{m}}{z^{m}}$. 注意到

$$
\sum_{m=1}^{\infty} m t^{m}=\frac{t}{(1-t)^{2}}, \quad|t|<1,
$$

得到

$$
\left\|x_{z}\right\|^{2}=\sum_{m=1}^{\infty}\left|x_{m}\right|^{2}=\frac{\left(|z|^{2}-1\right)^{2}}{|z|^{2}} \sum_{m=1}^{\infty} \frac{m}{\left(|z|^{2}\right)^{m}}=1 .
$$

固定 $m$, 当 $|z| \rightarrow 1_{+}$时 $x_{m} \rightarrow 0$, 因而当 $|z| \rightarrow 1_{+}$时 $\left\{x_{z}\right\}$ 弱收玫到 0 . 由于 $G(f)$ 是紧 的, 当 $|z| \rightarrow 1_{+}$时 $G(f) x_{z} \rightarrow 0$, 因此当 $|z| \rightarrow 1_{+}$时, $\left\langle G(f) x_{z}, \bar{x}_{z}\right\rangle \rightarrow 0$. 另一方面, 由 (1.5) 和 (4.2) 式可以得到

$$
\begin{aligned}
\left\langle G(f) x_{z}, \bar{x}_{z}\right\rangle & =\frac{\left(|z|^{2}-1\right)^{2}}{|z|^{2}} \sum_{m, n=1}^{\infty} \sqrt{m n} \alpha_{m n}(f) \frac{\sqrt{m}}{z^{m}} \frac{\sqrt{n}}{z^{n}} \\
& =\sum_{m, n=1}^{\infty} m n \alpha_{m n}(f) z^{-(m+n+2)}(z /|z|)^{2}\left(|z|^{2}-1\right)^{2} \\
& =-\frac{z^{2}}{6|z|^{2}} S(f)(z)\left(|z|^{2}-1\right)^{2},
\end{aligned}
$$

因此, 当 $|z| \rightarrow 1_{+}$时 $S(f)(z)\left(|z|^{2}-1\right)^{2} \rightarrow 0$, 即 $S(f) \in B_{0}$.

现在假设 $f \in \cup_{k<1} \Sigma(k)$ 是渐近共形的. 需要证明 $G(f): l^{2} \rightarrow l^{2}$ 是紧的. 利用定理 1 可以用逼近的方法来证明. 由于 $f$ 是渐近共形的, $f$ 可以拟共形延拓到单位圆 $\Delta$ 内, 使得 当 $|z| \rightarrow 1_{-}$时, $f$ 的复特征 $\mu=\partial_{\bar{z}} f / \partial_{z} f$ 满足 $\mu(z) \rightarrow 0$. 对于 $0<r<1$, 定义 $\mu_{r}$, 使得 在 $\Delta_{r}=\{z:|z|<r\}$ 上, $\mu_{r}=\mu$, 而在 $\Delta-\Delta_{r}$ 上, $\mu_{r}=0$. 由于 $f_{\mu_{r}}$ 在 $\Delta_{r}^{*}$ 上是共形的, 
$f_{\mu_{r}}$ 属于 Weil-Petersson 类. 下面的定理 3 说明 $G\left(\left[\mu_{r}\right]\right)$ 是一个 Hilbert-Schmidt 算子, 从而 是紧算子. 另一方面, 当 $r \rightarrow 1_{-}$时 $\tau\left(\left[\mu_{r}\right],[\mu]\right) \rightarrow 0$, Grunsky 的全纯性说明, 当 $r \rightarrow 1_{-}$时 $G\left(\left[\mu_{r}\right]\right) \rightarrow G([\mu])$. 因此, $G(f)=G([\mu])$ 是紧的.

注 3 由对称关系 $\alpha_{m n}(f)=\alpha_{n m}(f)$ 知

$$
\|G(f)\|=\sup _{x \in l^{2}} \frac{\|G(f) x\|}{\|x\|}=\sup _{x \in l^{2}} \frac{|\langle G(f) x, \bar{x}\rangle|}{\|x\|^{2}} .
$$

当 $f$ 是渐近共形时, 根据 $G(f): l^{2} \rightarrow l^{2}$ 的紧性, 容易看到存在非零的 $x \in l^{2}$, 使得 $G(f) x=$ $\|G(f)\| \bar{x}$, 且它同时达到上面的两个上确界.

注 4 在定理 2 的证明过程中用到了下面的定理 3. 事实上, 本文的最初出发点是讨 论 Grunsky 算子的紧性并证明定理 2 和 3. 当完成这些主要工作后, 作者得知 TakhtajanTeo (见文献 [30]) 已经用其他的方法证明了定理 3 的一个等价结果. 他们也证明了一个类似于 定理 2 的紧性结果.

定理 3 对于 $f \in \cup_{k<1} \Sigma(k), G(f): l^{2} \rightarrow l^{2}$ 是一个 Hilbert-Schmidt 算子当且仅当 $f$ 属 于 Weil-Petersson 类.

正如注 4 中提到的, Takhtajan-Teo ${ }^{[30]}$ 得到了定理 3 的一个等价结果. 在本节的余下部分, 将通过初等和直接的计算来给出我们的证明. 首先, 根据定义, $G(f)$ 是一个 Hilbert-Schmidt 算子是指 $\sum_{k=1}^{\infty}\left\|G(f) e_{k}\right\|^{2}<\infty$. 由于

$$
\sum_{k=1}^{\infty}\left\|G(f) e_{k}\right\|^{2}=\sum_{k, l=1}^{\infty}\left|\left\langle G(f) e_{k}, e_{l}\right\rangle\right|^{2}=\sum_{k, l=1}^{\infty} k l\left|\alpha_{k l}(f)\right|^{2}=\operatorname{Tr} G(f) \bar{G}(f),
$$

因此 $G(f)$ 是一个 Hilbert-Schmidt 算子当且仅当 $\operatorname{Tr} G(f) \bar{G}(f)<\infty$.

我们开始定理 3 的证明.

引理 4.1 如果 $\phi(z)=\sum_{n=2}^{\infty} a_{n} z^{-(n+2)}, \psi(z)=\sum_{n=2}^{\infty} b_{n} z^{-(n+2)}$, 则

$$
\langle\phi, \psi\rangle=2 \pi \sum_{n=2}^{\infty}\left(n^{3}-n\right)^{-1} a_{n} \bar{b}_{n} .
$$

证明 该引理由以下计算直接得到,

$$
\begin{aligned}
\langle\phi, \psi\rangle & =\iint_{\Delta^{*}} \phi(z) \bar{\psi}(z)\left(|z|^{2}-1\right)^{2} d x d y \\
& =\iint_{\Delta^{*}} \sum_{m, n=2}^{\infty} a_{m} \bar{b}_{n} z^{-(m+2)} \bar{z}^{-(n+2)}\left(|z|^{2}-1\right)^{2} d x d y \\
& =\sum_{m, n=2}^{\infty} a_{m} \bar{b}_{n} \iint_{\Delta^{*}} z^{-(m+2)} \bar{z}^{-(n+2)}\left(|z|^{2}-1\right)^{2} d x d y \\
& =2 \pi \sum_{n=2}^{\infty}\left(n^{3}-n\right)^{-1} a_{n} \bar{b}_{n} .
\end{aligned}
$$

定理 3 的必要性部分由以下引理直接得到.

引理 4.2 设 $f \in \cup_{k<1} \Sigma(k)$. 如果 $G(f): l^{2} \rightarrow l^{2}$ 是一个 Hilbert-Schmidt 算子, 则 $S(f) \in B_{2}$. 进一步地, $\|S(f)\|_{2}^{2} \leqslant 12 \pi \operatorname{Tr} G(f) \bar{G}(f)$.

证明 根据 (4.2) 式,

$$
S(f)(z)=-6 \sum_{m, n=1}^{\infty} m n \alpha_{m n}(f) z^{-(m+n+2)}=-6 \sum_{k=2}^{\infty}\left(\sum_{m+n=k} m n \alpha_{m n}(f)\right) z^{-(k+2)} .
$$


由引理 4.1 和 Schwarz 不等式,

$$
\begin{aligned}
\|S(f)\|_{2}^{2} & =72 \pi \sum_{k=2}^{\infty}\left(k^{3}-k\right)^{-1}\left|\sum_{m+n=k} m n \alpha_{m n}(f)\right|^{2} \\
& \leqslant 72 \pi \sum_{k=2}^{\infty}\left(k^{3}-k\right)^{-1} \sum_{m+n=k} m n \sum_{m+n=k} m n\left|\alpha_{m n}(f)\right|^{2} \\
& =12 \pi \sum_{m, n=1}^{\infty} m n\left|\alpha_{m n}(f)\right|^{2}=12 \pi \operatorname{Tr} G(f) \bar{G}(f) .
\end{aligned}
$$

接着证明定理 3 的充分性部分. 类似于引理 4.1 的证明可以得到

引理 4.3 如果 $\phi(z)=\sum_{n=1}^{\infty} a_{n} z^{-(n+1)}$, 则

$$
\iint_{\Delta^{*}}|\phi(z)|^{2} d x d y=\pi \sum_{n=1}^{\infty} n^{-1}\left|a_{n}\right|^{2} .
$$

现在假设 $\mu \in M_{2}$. 为简单起见, 记 $f=f_{\mu}$. 需要证明 $\operatorname{Tr} G(f) \bar{G}(f)<\infty$. 记

(4.1) 式说明

$$
S(f, z, \zeta)=\frac{f^{\prime}(z) f^{\prime}(\zeta)}{[f(z)-f(\zeta)]^{2}}-\frac{1}{(z-\zeta)^{2}} .
$$

$$
\begin{array}{rl}
\iint_{\Delta^{*}} & S(f, z, \zeta) \bar{\zeta}^{-(k+1)} d \xi d \eta \\
& =-\sum_{m, n=1}^{\infty} m n \alpha_{m n}(f) z^{-(m+1)} \iint_{\Delta^{*}} \zeta^{-(n+1)} \bar{\zeta}^{-(k+1)} d \xi d \eta \\
& =-\pi \sum_{m=1}^{\infty} m \alpha_{m k}(f) z^{-(m+1)} .
\end{array}
$$

引理 4.3 说明

$$
\iint_{\Delta^{*}}\left|\iint_{\Delta^{*}} S(f, z, \zeta) \bar{\zeta}^{-(k+1)} d \xi d \eta\right|^{2} d x d y=\pi^{3} \sum_{m=1}^{\infty} m\left|\alpha_{m k}(f)\right|^{2}
$$

因此,

$$
\begin{aligned}
\operatorname{Tr} G(f) \bar{G}(f) & =\sum_{m, k=1}^{\infty} m k\left|\alpha_{m k}(f)\right|^{2} \\
& =\frac{1}{\pi^{3}} \sum_{k=1}^{\infty} k \iint_{\Delta^{*}}\left|\iint_{\Delta^{*}} S(f, z, \zeta) \bar{\zeta}^{-(k+1)} d \xi d \eta\right|^{2} d x d y
\end{aligned}
$$

为证明 $\operatorname{Tr} G(f) \bar{G}(f)<\infty$, 我们来估计 $\iint_{\Delta^{*}} S(f, z, \zeta) \bar{\zeta}^{-(k+1)} d \xi d \eta$. 根据 Green 公式,

$$
\begin{array}{rl}
\iint_{\Delta^{*}} & S(f, z, \zeta) \bar{\zeta}^{-(k+1)} d \xi d \eta \\
& =\iint_{\Delta^{*}}\left(\frac{f^{\prime}(z) f^{\prime}(\zeta)}{[f(z)-f(\zeta)]^{2}}-\frac{1}{(z-\zeta)^{2}}\right) \bar{\zeta}^{-(k+1)} d \xi d \eta \\
& =-\frac{1}{2 i} \int_{\partial \Delta^{*}}\left(\frac{f^{\prime}(z)}{f(z)-f(\zeta)}-\frac{1}{z-\zeta}\right) \bar{\zeta}^{-(k+1)} d \bar{\zeta} \\
& =\frac{1}{2 i} \int_{\partial \Delta}\left(\frac{f^{\prime}(z)}{f(z)-f(\zeta)}-\frac{1}{z-\zeta}\right) \bar{\zeta}^{-(k+1)} d \bar{\zeta} \\
& =-\frac{1}{2 i} \int_{\partial \Delta}\left(\frac{f^{\prime}(z)}{f(z)-f(\zeta)}-\frac{1}{z-\zeta}\right) \zeta^{k-1} d \zeta
\end{array}
$$




$$
\begin{aligned}
& =-\frac{1}{2 i} \int_{\partial \Delta} \frac{f^{\prime}(z)}{f(z)-f(\zeta)} \zeta^{k-1} d \zeta \\
& =-\iint_{\Delta} \frac{f^{\prime}(z) \bar{\partial} f(\zeta) \zeta^{k-1}}{[f(z)-f(\zeta)]^{2}} d \xi d \eta .
\end{aligned}
$$

记 $g=f^{-1}$, 由 (4.6) 式可以得到

$$
\begin{aligned}
\iint_{\Delta^{*}}\left|\iint_{\Delta^{*}} S(f, z, \zeta) \bar{\zeta}^{-(k+1)} d \xi d \eta\right|^{2} d x d y \\
=\iint_{\Delta^{*}}\left|\iint_{\Delta} \frac{f^{\prime}(z) \bar{\partial} f(\zeta) \zeta^{k-1}}{[f(z)-f(\zeta)]^{2}} d \xi d \eta\right|^{2} d x d y \\
=\iint_{\Delta^{*}}\left|\iint_{f(\Delta)} \frac{f^{\prime}(z) \bar{\partial} g(\zeta) g^{k-1}(\zeta)}{[f(z)-\zeta]^{2}} d \xi d \eta\right|^{2} d x d y \\
=\iint_{f\left(\Delta^{*}\right)}\left|\iint_{f(\Delta)} \frac{\bar{\partial} g(\zeta) g^{k-1}(\zeta)}{(w-\zeta)^{2}} d \xi d \eta\right|^{2} d u d v \\
=\iint_{f\left(\Delta^{*}\right)}\left|\iint_{\mathbb{C}} \frac{\bar{\partial} g(\zeta) g^{k-1}(\zeta)}{(w-\zeta)^{2}} d \xi d \eta\right|^{2} d u d v \\
\quad \leqslant \iint_{\mathbb{C}}\left|\iint_{\mathbb{C}} \frac{\bar{\partial} g(\zeta) g^{k-1}(\zeta)}{(w-\zeta)^{2}} d \xi d \eta\right|^{2} d u d v .
\end{aligned}
$$

现在应用 Hilbert 变换 $H$ 的一个基本性质. Hilbert 变换 $H$ 由如下 Cauchy 主值积分定义:

$$
H(\mu)(z)=-\frac{1}{\pi} \iint_{\mathbb{C}} \frac{\mu(\zeta)}{(\zeta-z)^{2}} d \xi d \eta .
$$

$H$ 是平面 $\mathbb{C}$ 上平方可积函数空间上的等距算子 ${ }^{[31]}$, 因此, 由 (4.7) 式可以得到

$$
\begin{aligned}
\iint_{\Delta^{*}} \mid & \left.\iint_{\Delta^{*}} S(f, z, \zeta) \bar{\zeta}^{-(k+1)} d \xi d \eta\right|^{2} d x d y \\
& \leqslant \pi^{2} \iint_{\mathbb{C}}\left|\bar{\partial} g(\zeta) g^{k-1}(\zeta)\right|^{2} d \xi d \eta \\
& =\pi^{2} \iint_{f(\Delta)}\left|\bar{\partial} g(\zeta) g^{k-1}(\zeta)\right|^{2} d \xi d \eta \\
& =\pi^{2} \iint_{\Delta}\left|\bar{\partial} f(\zeta) \zeta^{k-1}\right|^{2} d \xi d \eta \\
& \leqslant \pi^{2} \iint_{\Delta}|\mu(\zeta)|^{2}|\zeta|^{2 k-2} d \xi d \eta \iint_{\Delta}|\partial f(\zeta)|^{2} d \xi d \eta,
\end{aligned}
$$

其中最后的不等式由 Schwarz 不等式得到. 因此由 (4.5) 和 (4.8) 式可以得到

$$
\begin{aligned}
\operatorname{Tr} G(f) \bar{G}(f) & =\frac{1}{\pi^{3}} \sum_{k=1}^{\infty} k \iint_{\Delta^{*}}\left|\iint_{\Delta^{*}} S(f, z, \zeta) \bar{\zeta}^{-(k+1)} d \xi d \eta\right|^{2} d x d y \\
& \leqslant \frac{1}{\pi} \iint_{\Delta}|\partial f(\zeta)|^{2} d \xi d \eta \sum_{k=1}^{\infty} k \iint_{\Delta}|\mu(\zeta)|^{2}|\zeta|^{2 k-2} d \xi d \eta \\
& =\frac{1}{\pi} \iint_{\Delta}|\partial f(\zeta)|^{2} d \xi d \eta \iint_{\Delta}|\mu(\zeta)|^{2}\left(\sum_{k=1}^{\infty} k|\zeta|^{2 k-2}\right) d \xi d \eta
\end{aligned}
$$




$$
=\frac{1}{\pi} \iint_{\Delta}|\partial f(\zeta)|^{2} d \xi d \eta \iint_{\Delta}|\mu(\zeta)|^{2}\left(1-|\zeta|^{2}\right)^{-2} d \xi d \eta .
$$

这便完成了定理 3 的证明.

\section{5 无穷小意义下的紧性}

第 4 节讨论了 Grunsky 算子的紧性, 刻画了万有 Teichmüller 空间 $T$ 中的点 $[\mu]$, 使 得相应的 Grunsky 算子 $G([\mu])$ 是紧算子或 Hilbert-Schmidt 算子. 在这一节中, 我们将讨论 Grunsky 映射在无穷小意义下的紧性. 确切地, 将刻画 Teichmüller 空间 $T$ 的切空间中的点, 使得 Grunsky 映射 $G$ 在零点的微分在这些点处是紧算子或 Hilbert-Schmidt 算子.

首先证明

引理 5.1 任给 $L^{\infty}(\Delta)$ 中的两元素 $\nu_{1}$ 和 $\nu_{2},\left\langle d_{0} \Psi\left(\nu_{1}\right), d_{0} \Psi\left(\nu_{2}\right)\right\rangle=12 \pi \operatorname{Tr} d_{0} \tilde{G}\left(\nu_{1}\right) \overline{d_{0} \tilde{G}\left(\nu_{2}\right)}$. 证明 对于 $\nu \in L^{\infty}(\Delta)$, 记

$$
c_{k}(\nu)=\frac{1}{\pi} \iint_{\Delta} \nu(z) z^{k-2} d x d y, \quad k \geqslant 2 .
$$

注意到

$$
\frac{1}{(1-t)^{4}}=\frac{1}{6} \sum_{k=2}^{\infty}\left(k^{3}-k\right) t^{k-2}, \quad|t|<1
$$

从 (2.3) 和 (5.1) 式得到

$$
\begin{aligned}
d_{0} \Psi(\nu)(z) & =-\frac{6}{\pi} \iint_{\Delta} \frac{\nu(\zeta)}{(\zeta-z)^{4}} d \xi d \eta \\
& =-\frac{1}{\pi} \iint_{\Delta} \sum_{k=2}^{\infty}\left(k^{3}-k\right) \nu(\zeta) \zeta^{k-2} z^{-(k+2)} d \xi d \eta \\
& =-\sum_{k=2}^{\infty}\left(k^{3}-k\right) c_{k}(\nu) z^{-(k+2)} .
\end{aligned}
$$

引理 4.1 和 $(5.2)$ 式蕴涵

$$
\left\langle d_{0} \Psi\left(\nu_{1}\right), d_{0} \Psi\left(\nu_{2}\right)\right\rangle=2 \pi \sum_{k=2}^{\infty}\left(k^{3}-k\right) c_{k}\left(\nu_{1}\right) \bar{c}_{k}\left(\nu_{2}\right) .
$$

另一方面, 由 (3.2) 式可以得到

$$
\begin{aligned}
\operatorname{Tr} d_{0} \tilde{G}(\nu) \overline{d_{0} \tilde{G}(\nu)} & =\sum_{m, n=1}^{\infty} m n c_{m+n}\left(\nu_{1}\right) \bar{c}_{m+n}\left(\nu_{2}\right) \\
& =\sum_{k=2}^{\infty} \sum_{m+n=k} m n c_{k}\left(\nu_{1}\right) \bar{c}_{k}\left(\nu_{2}\right) \\
& =\frac{1}{6} \sum_{k=2}^{\infty}\left(k^{3}-k\right) c_{k}\left(\nu_{1}\right) \bar{c}_{k}\left(\nu_{2}\right),
\end{aligned}
$$

因此, $\left\langle d_{0} \Psi\left(\nu_{1}\right), d_{0} \Psi\left(\nu_{2}\right)\right\rangle=12 \pi \operatorname{Tr} d_{0} \tilde{G}\left(\nu_{1}\right) \overline{d_{0} \tilde{G}\left(\nu_{2}\right)}$.

引理 5.1 的一个直接推论是

定理 4 任给 $\nu \in L^{\infty}(\Delta), d_{0} G\left(d_{0} \Phi(\nu)\right): l^{2} \rightarrow l^{2}$ 是一个 Hilbert-Schmidt 算子当且仅当 $d_{0} \Psi(\nu) \in B_{2}$. 
证明 由于 $d_{0} G\left(d_{0} \Phi(\nu)\right)=d_{0} \tilde{G}(\nu)$, 定理 4 由关系式 $\left\|d_{0} \Psi(\nu)\right\|_{2}^{2}=12 \pi \operatorname{Tr} d_{0} \tilde{G}(\nu) \overline{d_{0} \tilde{G}(\nu)}$ 得到.

\section{最后证明}

定理 5 任给 $\nu \in L^{\infty}(\Delta), d_{0} G\left(d_{0} \Phi(\nu)\right): l^{2} \rightarrow l^{2}$ 是一个紧算子当且仅当 $d_{0} \Psi(\nu) \in B_{0}$.

证明 假设 $d_{0} G\left(d_{0} \Phi(\nu)\right): l^{2} \rightarrow l^{2}$ 是一个紧算子. 类似于定理 2 的证明, 对 $z \in \Delta^{*}$, 记 $x_{z}=\left(x_{m}\right)$, 其中 $x_{m}=\frac{|z|^{2}-1}{|z|} \cdot \frac{\sqrt{m}}{z^{m}}$, 则 $\left\|x_{z}\right\|=1$, 且当 $|z| \rightarrow 1_{+}$时, $\left\{x_{z}\right\}$ 弱收玫到 0 . 由于 $d_{0} \tilde{G}(\nu)=d_{0} G\left(d_{0} \Phi(\nu)\right)$ 是紧的, 当 $|z| \rightarrow 1_{+}$时, $d_{0} \tilde{G}(\nu) x_{z} \rightarrow 0$, 从而 $\left\langle d_{0} \tilde{G}(\nu) x_{z}, \bar{x}_{z}\right\rangle \rightarrow 0$.

另一方面, (3.3) 式蕴涵

$$
\phi_{x_{z}}(w)=\frac{1}{\pi} \frac{\left(|z|^{2}-1\right)^{2}}{|z|^{2}} \sum_{m, n=1}^{\infty} \sqrt{m n} \frac{\sqrt{m}}{z^{m}} \frac{\sqrt{n}}{z^{n}} w^{m+n-2}=\frac{1}{\pi} \frac{z^{2}}{|z|^{2}} \frac{\left(|z|^{2}-1\right)^{2}}{(w-z)^{4}} .
$$

由 (2.3) 和 (3.4) 式可以得到

$$
\left\langle d_{0} \tilde{G}(\nu) x_{z}, \bar{x}_{z}\right\rangle=\frac{1}{\pi} \frac{z^{2}}{|z|^{2}}\left(|z|^{2}-1\right)^{2} \iint_{\Delta} \frac{\nu(w)}{(w-z)^{4}} d u d v=-\frac{1}{6} \frac{z^{2}}{|z|^{2}}\left(|z|^{2}-1\right)^{2} d_{0} \Psi(\nu)(z) .
$$

因此, 当 $|z| \rightarrow 1_{+}$时, $\left(|z|^{2}-1\right)^{2} d_{0} \Psi(\nu)(z) \rightarrow 0$, 即 $d_{0} \Psi(\nu) \in B_{0}$.

现在假设 $d_{0} \Psi(\nu) \in B_{0}$. 需要证明 $d_{0} G\left(d_{0} \Phi(\nu)\right)$ 是一个紧算子. 类似于定理 2 的证明, 我 们用逼近的方法. 记 $\tilde{\nu}(z)=-1 / 2\left(1-|z|^{2}\right)^{2} d_{0} \Psi(\nu)\left(\bar{z}^{-1}\right) \bar{z}^{-4}$. Teichmüller 理论中一个基本的 结果说明 $d_{0} \Psi(\tilde{\nu})=d_{0} \Psi(\nu)$, 从而 $d_{0} \Phi(\tilde{\nu})=d_{0} \Phi(\nu)$ (见文献 [18]). 由于 $d_{0} \Psi(\nu) \in B_{0}$, 因此 $\tilde{\nu} \in L_{0}$. 对 $0<r<1$, 考虑 $\tilde{\nu}_{r}$, 其中在 $\Delta_{r}=\{z:|z|<r\}$ 上, $\tilde{\nu}_{r}=\tilde{\nu}$, 而在 $\Delta-\Delta_{r}$ 上, $\tilde{\nu}_{r}=0$. 显然, $\tilde{\nu}_{r} \in L_{2}, d_{0} \Psi\left(\tilde{\nu}_{r}\right) \in B_{2}$. 定理 4 说明 $d_{0} G\left(d_{0} \Phi\left(\tilde{\nu}_{r}\right)\right)$ 是一个 Hilbert-Schmidt 算子, 从而 是一个紧算子. 另一方面, 由于 $r \rightarrow 1_{-}$时 $\left\|\tilde{\nu}_{r}-\tilde{\nu}\right\|_{\infty} \rightarrow 0$, Grunsky 映射的全纯性说明, 当 $r \rightarrow 1_{-}$时, $d_{0} G\left(d_{0} \Phi\left(\tilde{\nu}_{r}\right)\right) \rightarrow d_{0} G\left(d_{0} \Phi(\tilde{\nu})\right)$. 因此, $d_{0} G\left(d_{0} \Phi(\nu)\right)=d_{0} G\left(d_{0} \Phi(\tilde{\nu})\right)$ 一个紧算子. 证 毕.

\section{参 考 文 献}

1 Pommerenke C. Univalent Functions. Vandenhoeck and Ruprecht, 1975

2 Krushkal S L. Invariant metrics on Teichmüller spaces and quasiconformal extendability of analytic functions. Ann Acad Sci Fenn, 10: 299-303 (1985)

3 Krushkal S L. The Grunsky coefficient conditions. Siberian Math J, 28: 104-110 (1987)

4 Krushkal S L. Grunsky coefficient inequalities, Carathéodory metric and extremal quasiconformal mappings. Comment Math Helv, 64: 650-660 (1989)

5 Krushkal S L. Strengthening pseudoconvexity of finite-dimensional Teichmüller spaces. Math Ann, 290: 681-687 (1991)

6 Krushkal S L. Quasiconformal extremals of non-regular functionals. Ann Acad Sci Fenn, 17: 295-306 (1992)

7 Krushkal S L. Polygonal quasiconformal maps and Grunsky inequalities. J Anal Math, 90: 175-196 (2003)

8 Krushkal S L. Complex geometry of the universal Teichmüller space. Siberian Math J, 45: 646-668 (2004)

9 Krushkal S L. Grunsky inequalities of higher rank with applications to complex geometry and function theory. Contemp Math, 364: 127-153 (2004)

10 Krushkal S L. Plurisubharmonic features of the Teichmüller metric. Publ Inst Math, Nouv Ser, 75(89): 119-138 (2004)

11 Krushkal S L. The Schwarzian derivative and complex Finsler metrics. Contemp Math, 382: 243-262 $(2005)$ 
12 Shiga H. On analytic and geometric properties of Teichmüller spaces. J Math Kyoto Univ, 24: 441-452 (1984)

13 Shiga H. Characterization of quasi-disks and Teichmüller spaces. Tohoku Math J, 37: 541-552 (1985)

14 Shiga H, Tanigawa H. Grunsky's inequality and its applications to Teichmüller spaces. Kodai Math J, 16: 361-378 (1993)

15 Zuravlev I V. Univalent functions and Teichmüller spaces. Soviet Math Dokl, 21: 252-255 (1980)

16 Gardiner F P, Lakic N. Quasiconformal Teichmüller Theory. Math Surveys Monogr, 76, Providence, RI: Amer Math Soc, 2000

17 Lehto O. Univalent Functions and Teichmüller Spaces. New York: Springer-Verlag, 1986

18 Nag S. The Complex Analytic Theory of Teichmüller Spaces. London: Wiley-Interscience, 1988

19 Pommerenke C. Boundary Behaviour of Conformal Maps. Berlin: Springer-Verlag, 1992

20 Earle C J, Gardiner F P, Lakic N. Asymptotic Teichmüller space, Part I: The complex structure. Contemp Math, 256: 17-38 (2000)

21 Earle C J, Gardiner F P, Lakic N. Asymptotic Teichmüller space, Part II: The metric structure. Contemp Math, 355: 187-219 (2004)

22 Earle C J, Markovic V, Saric D. Barycentric extension and the Bers embedding for asymptotic Teichmüller space. Contemp Math, 311: 87-105 (2002)

23 Gardiner F P, Sullivan D. Symmetric structures on a closed curve. Amer J Math, 114: 683-736 (1992)

24 Cui G. Integrable asymptotic affine homeomorphisms of the circle and Teichmüller spaces. Sci China Ser A-Math, 43: 267-279 (2000)

25 Chae S B. Holomorphy and Calculus in Normed Spaces. New York: Marcel Dekker, 1985

26 Dineen S. Complex Analysis on Infinite Dimensional Spaces. London: Springer-Verlag, 1999

27 Dunford N. Uniformity in linear spaces. Tran Amer Math Soc, 44: 305-356 (1938)

28 Earle C J. The holomorphic contractibility of two generalized Teichmüller spaces. Publ Inst Math, Nouv Ser, 75: 109-117 (2004)

29 Kaup L, Kaup B. Holomorphic functions of several variables. Gruyter Studies in Math, 1983, 3

30 Takhtajan L, Teo L. Weil-Petersson metric on the universal Teichmüller space. Mem Amer Math Soc, 183(861): 1-119 (2006)

31 Ahlfors L V. Lectures on Quasiconformal Mappings. Princeton: Van Nostrand Company, 1966 